\title{
Autoconcepto y Talento: Una Relación que Favorece el Logro Académico
}

\section{Self-Concept and Talent: A Connection That Promotes Academic Achievement}

\author{
María de la Luz González y Daniella Leal \\ Pontificia Universidad Católica de Chile
}

\author{
Carolina Segovia \\ Centro de Estudios Públicos
}

\author{
Violeta Arancibia \\ Pontificia Universidad Católica de Chile
}

\begin{abstract}
En este artículo se presentan los resultados de un estudio cuyo objetivo fue estimar la asociación entre autoconcepto y talento académico con los resultados académicos en lenguaje y matemática de alumnos de contextos socioeconómicos vulnerables. Se utilizó una metodología cuantitativa con un diseño correlacional y una muestra intencionada. Se recolectaron los datos a través de pruebas alineadas al currículum (Sistema de Evaluación del Progreso en el Aprendizaje [SEPA]), un test de inteligencia práctica, creativa y analítica y un cuestionario de autoconcepto académico, aplicados a 1411 alumnos, y un cuestionario aplicado a 1411 apoderados. Los resultados, obtenidos por medio de $t$ de Student y modelos de regresión múltiple, apoyan la asociación entre expectativas académicas, autoconcepto y talento académico y sugieren que estos 3 elementos conforman un círculo virtuoso que permite a los alumnos obtener buenos resultados académicos.
\end{abstract}

Palabras clave: talento académico, autoconcepto académico, logro académico

\begin{abstract}
This article presents the results of a study that estimates the association between self-esteem and academic talent, using the academic results for language and math of students in vulnerable socioeconomic conditions. A correlational design with quantitative methodology and an intentional sample were used. Data were collected through tests that follow the curriculum (Assessment System of Progress in Learning [SEPA]), a practical, creative and analytic intelligence test, a questionnaire on academic self-concept applied to 1411 students, and a questionnaire applied to 1411 parents and/or legal guardians. The results, obtained via the use of Student's $t$ and multiple regression models, support the association between academic expectations, self-concept, and academic talent and suggest that these 3 elements form a virtuous cycle that fosters good academic performance.
\end{abstract}

Keywords: academic talent, academic self-concept, academic achievement

Los niños talentosos se destacan por sus capacidades cognitivas, como la memoria, los procesos autorreguladores, la velocidad de aprendizaje, la flexibilidad cognitiva y la preferencia por la complejidad (Shore \& Kanevsky, 1993), y son estas capacidades las que aumentan la probabilidad de obtener mejores resultados académicos.

$\mathrm{Al}$ entender el autoconcepto como un "poderoso sistema de estructuras cognitivas que tiene una alta probabilidad de mediar las interpretaciones y respuestas a eventos y comportamientos que involucren o estén dirigidos al individuo" [Traducción de las autoras] (Nurius, 1986, p.

María de la Luz González L. y Daniella Leal V., Instituto de Sociología, Pontificia Universidad Católica de Chile, Santiago, Chile; Carolina Segovia A., Centro de Estudios Públicos, Santiago, Chile; Violeta Arancibia C., Escuela de Psicología, Pontificia Universidad Católica de Chile, Santiago, Chile.

La investigación fue financiada con fondos del Consejo Nacional de Ciencias y Tecnología de Chile (Proyecto FONDEF D05I10398) y la Corporación de Educación de Puente Alto.

La correspondencia relativa a este artículo debe ser dirigida a María de la Luz González L., Instituto de Sociología, Pontificia Universidad Católica de Chile, Vicuña Mackenna 4860, Macul, Santiago, Chile. E-mail: mlgonzal@uc.cl 
435), es posible comprender el rol que este sistema de estructuras tiene en los logros académicos de los estudiantes. Las actitudes y creencias frente al aprendizaje y las acciones y conductas en el sistema escolar estarán mediadas por el autoconcepto académico que el alumno presente.

Se ha observado que los estudiantes talentosos suelen presentar un autoconcepto más alto en áreas académicas (Colangelo, 2002), percepción que se basa y se refuerza en los resultados académicos que obtienen (Marsh \& Craven, 2006).

El objetivo del estudio que se reporta fue estimar la relación entre el autoconcepto y el talento académico con el rendimiento académico. Analizamos el rol que ejerce el autoconcepto alto en niños sin talentos académicos y lo comparamos con lo que sucede en el grupo de niños con talentos académicos. Las preguntas que guiaron esta investigación fueron: ¿Cuáles son los factores que influyen en el rendimiento académico de los alumnos? ¿Existen diferencias en estos factores entre el grupo de niños con talentos y el grupo de niños sin talentos?

La importancia de esta investigación radica en la posibilidad de indagar en la relación que se establece entre autoconcepto académico y talento académico en la consecución de logros académicos en contextos socioeconómicos vulnerables, con miras a realizar intervenciones que permitan trabajar el autoconcepto como un factor protector para el rendimiento académico, desarrollando estrategias diferenciadas para cada grupo de niños (con talento y sin talento académico).

\section{Talento Académico: Identificación y Características de los Individuos con Talentos}

Desde principios de siglo XX se han planteado variadas alternativas para identificar a los niños con talentos académicos, las cuales van desde el test que mide el coeficiente intelectual de Binet (Colangelo \& Davis, 2003) hasta modelos más funcionales que consideran la relación de variadas habilidades.

Desde la visión de Sternberg (1997), el talento académico es entendido en base a la interacción de tres habilidades: (a) habilidades analíticas para resolver problemas y juzgar la calidad de las ideas, las que corresponden a las habilidades de pensamiento lógico formal; (b) habilidades creativas para generar nuevos problemas, buenas ideas y plantear preguntas adecuadas para la búsqueda de soluciones y (c) habilidades prácticas para aplicar las ideas y soluciones para adecuarse a los distintos contextos y para resolver problemas de manera práctica. El mismo autor señala que un alto desempeño intelectual implica el uso integrado y la capacidad para coordinar estas tres habilidades. Otros modelos que consideran el talento académico como una interrelación de varias dimensiones son el modelo orientado al rendimiento de Renzulli (Renzulli \& Reis, 2003) y los modelos socioculturales de Gagné (2003) y Tannenbaum (2003). En el primer caso, la medición del talento académico considera la habilidad por encima de la media, el compromiso con la tarea y la creatividad. En el segundo caso, se amplía la visión al contexto donde interactúan factores individuales y sociales para la manifestación del talento académico.

El talento académico se asocia a la presencia de capacidades cognitivas sobresalientes como la memoria, la velocidad de aprendizaje, el conocimiento procedural, la capacidad de categorización, la flexibilidad cognitiva y la preferencia por la complejidad (Shore \& Kanevsky, 1993). Otros autores han incluido también la curiosidad intelectual, la creatividad y la capacidad de concentración entre las habilidades que presentan generalmente los sujetos con talentos académicos (Silverman, 1997).

En relación a las características socioemocionales de los niños y jóvenes con talentos académicos, se ha observado que estos presentan menos vulnerabilidad emocional (Porter, 2006), ya que sus altas habilidades cognitivas les permiten relacionarse adecuadamente con los estímulos emocionales. 
Varios autores han manifestado que los niños con talentos académicos tienden a buscar la compañía de niños con similar desarrollo intelectual (Davis, 1924; Hollingworth, 1931; O’Shea, 1960, todos citados en Bralic \& Romagnoli, 2000) y que se sienten poco aceptados por sus pares (George, 1992; Terrasier, 1998). Estudios empíricos han demostrado que los niños con mayores capacidades cognitivas son mejores para idear estrategias para resolver problemas sociales (Freeman, 2006), destacan en la capacidad de razonamiento relacionada a la comprensión y la definición del problema social, la planificación de estrategias y en la capacidad para anticipar consecuencias sociales de sus actos (López, 2007).

\section{Talento Académico: Características Familiares}

El contexto familiar donde se desenvuelve un niño o joven con talento académico presenta una clara asociación con los logros que estos niños pueden alcanzar. Así, se ha observado que las expectativas que tienen los padres sobre el desempeño académico de sus hijos tienen una relación significativa con la motivación que presentan los hijos hacia las actividades académicas y con sus éxitos académicos (Chan 2005; Robinson, Lanzi, Weinberg, Ramey \& Ramey, 2002).

Por otro lado, se ha explicitado que los recursos materiales de apoyo al estudiante al interior del hogar son claves para potenciar las habilidades de los niños, entregándoles mayores oportunidades para que desarrollen su potencial (Bloom, 1985).

Por último, algunos autores manifiestan que las familias de niños y jóvenes talentosos se caracterizan por tener padres con más años de escolaridad que los individuos no talentosos (Robinson et al., 2002), lo que potencia el aprendizaje del estudiante, al estar inserto en un medio donde se dispone de mayores recursos para el apoyo al aprendizaje.

\section{Autoconcepto: Definiciones y Mediciones}

Nurius (1986) define el autoconcepto como un sistema de estructuras cognitivas que media interpretaciones y respuestas de los individuos. El autoconcepto dirige la conducta de los individuos mediante el mecanismo de selección y procesamiento de la información y a través de la entrega de un marco interpretativo de las consecuencias de esas conductas (Núñez \& González, 1994). Por lo tanto, la información sobre el autoconcepto de los individuos presenta un alto potencial para generar un cambio relevante en las actitudes de las personas (Marsh, 2006).

Herbert W. Marsh (2006) ha realizado importantes contribuciones en torno al autoconcepto durante las últimas décadas, basándose en sus inicios en el modelo desarrollado por Shavelson, Hubner y Stanton en 1976. Este modelo considera el autoconcepto como la percepción de sí mismo que se desarrolla en base a las experiencias con el medio, por lo tanto, está especialmente influido por la evaluación de las personas cercanas, por los refuerzos y las atribuciones del propio comportamiento. Las percepciones que se hacen de sí mismo están conformadas por la información que es entregada por las personas que son significativas y que influyen fuertemente en los individuos, tales como la madre, los hermanos o los profesores (Sullivan, 1953, citado en Núñez \& González, 1994). El análisis del autoconcepto muestra la relevancia de la comparación social a través de una serie de estrategias de marcos de ajuste con otros pares (Marsh, 2006). Estas comparaciones tienen efectos directos sobre el autoconcepto académico, el cual, a su vez, se asocia con el desempeño de los estudiantes. Cuando estos desempeños caen, se produce un reforzamiento negativo del autoconcepto que posteriormente tendrá efectos aún más negativos sobre el desempeño.

Shavelson et al. (1976) dividen el autoconcepto en dos dimensiones: académico y no académico. El primero incluye a las diferentes áreas curriculares escolares y el segundo incluye el autoconcepto a nivel social, emocional y físico (Marsh, 2006). En base a lo anterior, Marsh genera el instrumento de medición del autoconcepto conocido como SDQ (Marsh, Debus \& 
Bornholt, 2005), con versiones distintas para las diferentes edades. Este cuestionario fue reconocido en 1996 por una comisión de la American Psychological Association como el instrumento más válido para la medición del autoconcepto. Pese a todas las bondades del instrumento y a pesar de que los niños tienen una adecuada comprensión de las dimensiones sobre las cuales son interrogados, los propios autores reconocen que las mediciones en niños entre cinco y ocho años son bastante débiles en relación a la coherencia general de la prueba.

En ese mismo sentido, se pone en duda la confiabilidad de los cuestionarios aplicados directamente a los niños (Arancibia, Maltes \& Álvarez, 1990), ya que los auto-informes no incorporarían en la determinación de los puntajes las bases sobre las cuales se realiza la valoración (Damon \& Hart, 1982). Por otro lado, las mediciones a través de entrevistas semiestructuradas tienen como limitante para determinar la capacidad de los niños el tener que confiar en la verbalización espontánea de ellos, la cual es posible que no contenga todos los elementos que estos realmente utilizan (Stipek \& Tannatt, 1984). Es por ello que se plantea la conveniencia de realizar la evaluación de autoconcepto mediante un observador externo (Demo, 1985).

El observador externo considerado como privilegiado para evaluar el autoconcepto de los niños es el profesor. Esta visión es respaldada con evidencia como la presentada por Brunel, Dupuy-Walker y Schleifer (1990), en la que se observa la gran capacidad predictiva de los profesores al momento de evaluar el autoconcepto académico de sus estudiantes con relación al desempeño efectivo.

Arancibia et al. (1990) validaron el instrumento desarrollado por Purkey, Cage y Fahey (1973), conocido como Florida KEY, para realizar mediciones de autoconcepto académico en la población escolar chilena. La razón para elegir este instrumento -que es el que se empleó en esta investigación- es evitar los problemas derivados del autoinforme. Al emplearse un instrumento que está basado en observaciones previas a niños con distintos niveles de autoconcepto, se cuenta con una información más global de la realidad del niño.

\section{La Relación Entre Autoconcepto y Talento Académico}

Los estudiantes talentosos presentan un mejor concepto de sí mismo en áreas académicas que en otras áreas (Colangelo \& Assouline, 1995, 2000, citados en Colangelo, 2002), el que se caracteriza por una actitud desafiante frente a la tarea, capacidad para correr riesgos, buena disposición frente a tareas difíciles o complejas y exactitud para evaluar su funcionamiento o desempeño (Neihart, Reis, Robinson \& Moon, 2002). Así, presentan una autoestima más alta que sus pares, pero muchas veces basada únicamente en sus éxitos académicos.

Una mayor autoestima genera mayor creatividad y ayuda a postergar una gratificación inmediata (Freeman, 2006), lo que, a su vez, generaría mayores logros a nivel académico. El hecho de que los niños académicamente talentosos presenten un autoconcepto positivo y mayor que sus pares se ve potenciado por un desempeño de carácter impecable en lo académico (Marsh \& Craven, 2006). Este desempeño refuerza el autoconcepto del estudiante, generándose un círculo virtuoso. Cuando se obtienen buenos resultados académicos se genera un mayor interés y motivación para incrementar los conocimientos, lo que contribuye al uso de estrategias de aprendizaje significativo, colaborando con los éxitos académicos (Valle, González, Núñez, Rodríguez \& Piñeiro, 1999).

El autoconcepto funciona, entonces, como mediador para la obtención de resultados deseables, es decir, tiene un efecto de reforzamiento. De ahí la importancia de la formulación y potenciamiento de ideas positivas para mejorar el desempeño efectivo de los individuos (Marsh \& Craven, 2006). Lo anterior es particularmente relevante para los estudiantes talentosos, puesto que permite entender el vínculo entre imágenes positivas de sí mismo y buen desempeño y, a su vez, la importancia de mantener desempeños satisfactorios que sirvan como incentivos a su autoconcepto académico. 
De esta manera, el autoconcepto se convierte en el vehículo a través del cual se pueden actualizar las potencialidades de los estudiantes (Cross \& Swiatek, 2009; Morawska \& Sanders, 2009), razón por la cual es relevante la mantención de un autoconcepto positivo con el fin de aprovechar todo el potencial de los individuos y evitar, así, el desperdicio de capacidades que finalmente se traduce en una pérdida social (Marsh, 2006).

Con el objetivo de comprender el rol del autoconcepto académico y el talento académico en la obtención de logros académicos realizamos un estudio correlacional. Las hipótesis que guiaron este estudio fueron que: (a) los niños con talentos académicos presentan un mayor autoconcepto que los niños sin talento académico y (b) los niños evaluados identificados con talentos académicos y con alto autoconcepto tienen mejores resultados académicos que los niños sin talentos académicos o con bajo autoconcepto.

\section{Método}

\section{Participantes}

Realizamos esta investigación en el contexto del programa piloto PENTA UC Escolar. Este es un programa para el desarrollo de talentos académicos realizado al interior de las escuelas, con currículum de enriquecimiento en lenguaje y matemáticas. Se diseñó para ser implementado en un contexto social vulnerable, por lo que se seleccionaron para el piloto 10 colegios municipales con mayor cantidad de alumnos de la comuna de Puente Alto y similares en cantidad de alumnos, profesores y en sus resultados académicos. De estos 10 colegios se asignaron al azar cinco colegios experimentales y cinco colegios control. Los datos que utilizamos en esta investigación correspondieron a 1.411 alumnos de $1^{\circ}$ a $4^{\circ}$ básico de los cinco colegios experimentales.

La comuna de Puente Alto, perteneciente a la Región Metropolitana, tiene 629.861 habitantes con un promedio de escolaridad de 10,7 años y de ingresos promedio de hogares de US $\$$ 1.288. Actualmente la comuna cuenta con un $10,6 \%$ de pobreza, un $1,9 \%$ de analfabetismo (Chile, Ministerio de Planificación, 2006) y 25.541 niños asisten a colegios administrados por la municipalidad, lo que corresponde al $23 \%$ de las matrículas de la comuna (Chile, Ministerio de Educación, 2008).

El 12\% (169 alumnos) de los alumnos de la muestra fue identificado como alumno con talento académico en base a tres pruebas: (a) Inteligencia Práctica, (b) Inteligencia Analítica y (c) Inteligencia Creativa. Estandarizamos el puntaje obtenido en cada una de las pruebas en relación al curso del alumno, sumando luego los puntajes $\mathrm{Z}$ de las tres pruebas y, nuevamente, transformando esta sumatoria en un puntaje Z. Luego establecimos para cada curso el puntaje equivalente al percentil 90. Basándonos en lo recomendado en la literatura revisada, identificamos como alumnos con talentos los que se encontraban sobre el percentil 90 en cada curso (Benito, 1996).

El $48 \%$ de los alumnos de la muestra son hombres y el $52 \%$, mujeres. El $42 \%$ de los alumnos identificados como alumnos con talento académico son mujeres y el 58\%, hombres.

\section{Instrumentos}

Sistema de Evaluación del Progreso en el Aprendizaje (SEPA) de Lenguaje y Matemáticas. Estas pruebas nos permitieron evaluar el rendimiento académico de los alumnos pertenecientes a la muestra. Estas están alineadas al currículum nacional en las áreas de lenguaje y matemáticas y fueron elaboradas especialmente para el proyecto piloto PENTA UC Escolar por MIDE UC. En los análisis utilizamos el puntaje estandarizado obtenido por cada estudiante en lenguaje y matemáticas. Este puntaje tiene una media de 50 puntos y una desviación estándar de 10 puntos. 
Test de Inteligencia Práctica. Este test fue diseñado especialmente para el programa PENTA UC escolar (Preiss, Arancibia, Rosas \& García, 2010), siendo el primero en medir este tipo de habilidades para estudiantes de primer ciclo básico en el contexto chileno (Preiss, Arancibia, Muñoz et al., 2010a). Está conformado por 25 ítems para $1^{\circ}$ y $2^{\circ}$ básico y 27 para $3^{\circ}$ y $4^{\circ}$ básico. Cada ítem consiste en la exposición de una situación que plantea un problema cotidiano para los niños, entregando tres alternativas de respuesta. En cada ítem la situación y las alternativas se encuentran en texto y en dibujos.

En su versión para $1^{\circ}$ y $2^{\circ}$ básico este test muestra un nivel de confiabilidad de 0,73 y en su versión para $3^{\circ}$ y $4^{\circ}$ básico, de 0,78 . El indicador utilizado en esta ocasión para medir la confiabilidad fue K-R 20, el cual se utiliza habitualmente con ítems cuya puntuación se transforma en una variable dicotómica (que indica respuestas correctas o incorrectas). Los valores sobre 0,7 en este indicador son considerados aceptables.

Test de Inteligencia Creativa. Este test es utilizado por Sternberg (1997) en el PACE Center para medir creatividad. En este se solicita al sujeto evaluado que desarrolle un producto (con calcomanías) que resuelva un problema novedosamente. En la versión adaptada al contexto chileno y para el programa piloto PENTA UC Escolar se les solicita a los alumnos la realización de un juguete y la asignación de un nombre para el mismo. A los alumnos de $3^{\circ}$ y $4^{\circ}$ básico se les pide, además, que expliquen su funcionamiento (Preiss, Arancibia, Rosas et al., 2010). Los productos elaborados por los estudiantes son evaluados por, al menos, dos jueces, en base a una pauta definida previamente (Preiss, Arancibia, Muñoz et al., 2010b). La confiabilidad de las puntuaciones se mide a través del nivel de acuerdo que existe en los puntajes asignados por los jueces y mediante una correlación de Pearson entre los valores asignados por ellos. Valores superiores a 0,75 en ambos casos son considerados aceptables. Los resultados mostraron que los niveles de acuerdo entre los jueces superaron el $75 \%$ y se obtuvo una correlación de Pearson, entre los puntajes asignados por los jueces, de 0,80 en la medición realizada para la evaluación de impacto del programa piloto PENTA UC Escolar.

Test de Inteligencia Analítica. Este test está basado en la estructura de inteligencia del modelo de Berlín (TEBID) y fue adaptado para el primer ciclo básico por investigadores del Centro de Estudios y Desarrollo de Talentos de la Pontificia Universidad Católica de Chile (Preiss, Arancibia, Rosas et al., 2010). Es un test de estructura factorial que mide memoria, procesos deductivos e inductivos y creatividad en las áreas de lenguaje y matemáticas. La versión para $1^{\circ}$ y $2^{\circ}$ básico consiste en un cuadernillo de 34 páginas, con 10 subpruebas. Cada subprueba mide diferentes habilidades: creatividad verbal, rapidez verbal — numérica y figural—, memoria verbal — numérica y figural—, profundidad figural y verbal y proceso numérico. El test presenta una consistencia interna, estimada a través de alfa de Cronbach, de 0,73. La versión para $3^{\circ}$ y $4^{\circ}$ básico consiste en un cuadernillo de 40 páginas, con 12 subpruebas. Cada subprueba mide diferentes habilidades: creatividad verbal — numérica y figural—, rapidez verbal —numérica y figural—, memoria verbal —numérica y figural—, profundidad figural y verbal y proceso numérico. Presenta una consistencia interna, estimada a través de alfa de Cronbach, de 0,69. Las muestras usadas para estos cálculos son las de los estudiantes de colegios experimentales en 2007.

Test de Autoconcepto Académico (TAC). El autoconcepto académico lo evaluamos a través de un test basado en el reporte de los profesores sobre sus alumnos. El test que utilizamos en esta investigación es una versión estandarizada para Chile (Arancibia et al., 1990) del test Florida KEY (Purkey et al., 1973). Este está compuesto por cuatro dimensiones:

1. Relaciones con otros. Esta dimensión, identificada como la de mayor relevancia, abarca los puntajes relacionados con los niveles de confianza y aprecio que el estudiante mantiene con otras personas. Un alto puntaje en esta dimensión se vincula a la sensación de pertenencia de los estudiantes. Esta dimensión está compuesta por los siguientes indicadores: (a) Se 
lleva bien con otros profesores, (b) Dice la verdad acerca de su trabajo escolar, (c) Dice cosas buenas de su escuela y (d) Se lleva bien con sus compañeros.

2. Asertividad. Esta dimensión puede ser descrita como aquella referida a las acciones personales que le permiten al individuo actuar de acuerdo a sus propios intereses, sin inducir ansiedad y expresando honestamente los propios sentimientos. Un alto puntaje en esta dimensión se refiere a la disposición a no ceder en pos de lo que se considera justo. Esta dimensión está compuesta por los siguientes indicadores: (a) Se ofrece para responder preguntas en clases, (b) Hace preguntas atingentes en clases, (c) Se ofrece para hablar frente al curso, (d) Expresa claramente sus propias ideas y (e) Muestra interés en ser un líder.

3. Compromiso. Esta dimensión señala la confianza en el propio potencial. Altos puntajes en esta dimensión indican mayor disposición al riesgo. Esta dimensión está compuesta por los siguientes indicadores: (a) Dedica tiempo ayudando a otros, (b) Inicia nuevas actividades escolares, (c) Muestra interés en el trabajo escolar de los demás, (d) Se le ocurren cosas nuevas que hacer en la escuela, (e) Se ofrece para hacer trabajos adicionales en la sala, (f) Participa activamente en la escuela y (g) Habla con sus profesores acerca de cosas personales.

4. Enfrentamiento a situaciones escolares. Esta dimensión está planteada como un indicador de confianza en la propia habilidad académica. El estudiante con puntaje alto en esta dimensión demuestra involucramiento e interés por lo que ocurre en la sala de clases. Esta dimensión está compuesta por los siguientes indicadores: (a) Es perseverante en su trabajo escolar, (b) Hace sus tareas con cuidado, (c) Presta atención en clases, (d) Termina sus tareas escolares y (e) Se muestra seguro de su trabajo escolar.

Todos los indicadores se evalúan en una escala de 1 a 6 , siendo 1 nunca y 6 casi siempre. La consistencia interna del test de autoconcepto fue estimada, a través de alfa de Cronbach, en 0,95. La consistencia interna por factor fue estimada en 0,95 para el Factor 1, 0,92 para el Factor 2, 0,91 para el Factor 3 y 0,84 para el Factor 4.

Cuestionario de Apoderados. En el análisis incluimos variables del contexto familiar con el propósito de controlar el efecto del autoconcepto académico y el talento académico sobre el logro académico. Según los antecedentes bibliográficos revisados, estas variables muestran una influencia sobre el logro académico (Bloom, 1985; Chan 2005; Robinson et al., 2002). Esta información la obtuvimos a través de la aplicación de un cuestionario administrado a los padres y apoderados de los estudiantes incluidos en la muestra. Este cuestionario fue elaborado por el equipo de investigadores del programa piloto PENTA UC Escolar para la evaluación de impacto del programa.

Las variables consideradas fueron:

- Nivel educacional de los padres. Nivel educacional alcanzado por el padre/padrastro y por la madre/madrastra del niño. Se mide en 10 categorías que van desde sin educación hasta educación universitaria completa o más. El nivel educacional del padre y de la madre lo analizamos de forma dicotómica, identificando dos grupos: padres con educación media o superior y padres con educación hasta media incompleta.

- Expectativas académicas sobre los hijos. Esta variable considera ocho categorías, desde no terminará la enseñanza básica hasta completará un postgrado en la universidad. Las expectativas académicas también las tratamos de forma dicotómica: padres que tienen para sus hijos expectativas de estudios superiores y padres con expectativas hasta educación media o menos.

- Tenencia de computador. Variable dicotómica (si o no).

- Tenencia de un espacio para estudiar. Variable dicotómica (sí o no).

Las pruebas SEPA y los test de inteligencia analítica, práctica y creativa fueron revisados y puntuados de acuerdo a los procedimientos propuestos por cada uno de sus autores. 
Las respuestas de los cuestionarios a padres y del test de autoconcepto fueron digitadas y procesadas a través del programa SPSS 15.

\section{Procedimiento}

Los test de autoconcepto fueron respondidos por profesores durante el año 2007 en las dependencias de cada uno de los colegios. Los profesores no tenían conocimiento de los resultados de la selección para el programa, por lo tanto, no sabían si su alumno estaba identificado o no como un alumno con talentos académicos.

Las pruebas de inteligencia práctica, creativa y analítica y las SEPA fueron aplicadas a los alumnos durante el año 2007 en las dependencias de cada uno de los colegios durante su jornada escolar. El consentimiento informado fue entregado por la corporación municipal, organismo responsable de cada colegio. En este consentimiento se hacía referencia a la confidencialidad de los datos de los participantes.

Los datos de los apoderados fueron recogidos durante el año 2007 en las dependencias de cada uno de los colegios durante una reunión de apoderados. Los apoderados no tenían conocimiento de los resultados de la selección para el programa, por lo tanto, no sabían si su hijo estaba identificado o no como un alumno con talentos académicos.

\section{Plan de Análisis}

Basamos el análisis de resultados en la distinción fundamental entre alumno con talento académico y alumno sin talento académico; a partir de esto, y bajo la hipótesis de que el autoconcepto es significativamente diferente entre ellos, testeamos los niveles de autoconcepto en sus distintas dimensiones y en el indicador final. Es así que en la primera sección de resultados exponemos la relación entre autoconcepto y talento académico, utilizando test de diferencia de medias para muestras independientes.

Más allá de evaluar si existen diferencias en el autoconcepto entre niños con talento y sin talento, también nos interesaba explorar si esta asociación se mantenía en presencia de ciertas variables estructurales y contextuales y si ellas podían afectar el rendimiento académico. De esta manera, estimamos diversos modelos de regresión múltiple, cuyo objetivo fue someter a prueba esta relación y ver su asociación con el rendimiento. En estos modelos incluimos como variables dependientes el rendimiento en matemática y lenguaje, por medio del puntaje en la prueba SEPA en estas mismas materias. En los modelos integramos variables independientes en bloque en base a su naturaleza. En el primer bloque consideramos únicamente la variable talento, en el segundo bloque integramos variables de la educación de los padres y en el tercer bloque integramos las expectativas de los padres. Luego estimamos modelos integrando los recursos en el hogar, el sexo y, finalmente, el autoconcepto.

\section{Resultados}

\section{Asociación Entre Talento y Autoconcepto Académico}

En la Tabla 1 destacamos las diferencias en autoconcepto académico entre el grupo de niños identificados como talentosos y el grupo de niños no talentosos. El autoconcepto total y todas sus dimensiones evaluadas son más altas en niños identificados como talentosos académicamente, apoyando nuestra primera hipótesis: estudiantes con talentos académicos presentan un nivel más alto de autoconcepto académico. 
Tabla 1

Diferencia en Puntajes de Autoconcepto y sus Dimensiones, Según Condición de Talento

\begin{tabular}{llcc}
\hline \multicolumn{1}{c}{ Dimensión autoconcepto académico } & No talento & Talento \\
\hline Enfrentamiento & $M$ & 22,67 & 26,32 \\
& $D E$ & 6,23 & 4,59 \\
& $95 \%$ IC & {$[22,46,22,87]$} & {$[25,90,26,77]$} \\
Compromiso & & $t(644)=-14,95, p<0,001, d=-0,60$ \\
& $M$ & 27,67 & 32,13 \\
& $D E$ & 8,91 & 7,84 \\
Asertividad & $95 \%$ IC & {$[27,38,27,97]$} & {$[31,40,32,88]$} \\
& & $t(576)=-10,99, p<0,001, d=-0,51$ \\
& $M$ & 19,48 & 22,96 \\
Relaciones con otros & $D E$ & 6,82 & 6,28 \\
& $95 \%$ IC & {$[19,25,19,70]$} & {$[22,36,23,55]$} \\
& & $t(561)=-10,75, p<0,001, d=-0,51$ \\
Autoconcepto académico total & $M$ & 19,79 & 21,29 \\
& $D E$ & 4,06 & 3,30 \\
& $95 \%$ IC & {$[19,65,19,92]$} & {$[20,98,21,60]$} \\
& $M$ & $t(600)=-8,68, p<0,001, d=-0,38$ \\
& $D E$ & 89,34 & 102,50 \\
& $95 \%$ IC & {$[88,87,90,35]$} & {$[100,97,81$} \\
& & $t(596)=-13,42, p<0,001, d=-0,59$ \\
\hline
\end{tabular}

$d=d$ de Cohen

\section{Asociación Entre Rendimiento Académico, Autoconcepto y Talento}

En la Tabla 2 se observa una fuerte asociación del talento académico y las expectativas educacionales de los padres (educación superior), y una menor del hecho de tener un espacio donde estudiar, con el rendimiento en la prueba SEPA de Lenguaje (Modelos 4 y 5). Cuando se integra el puntaje de autoconcepto (Modelos 6 y 7), solo el talento y las expectativas educacionales mantienen una relación significativa con el rendimiento en lenguaje, explicando un 17\% de la varianza del puntaje de esta prueba. No se observan diferencias por sexo en la asociación entre el autoconcepto y los resultados de los alumnos en esta prueba (Modelo 7). Estos resultados también apoyan nuestra hipótesis sobre la asociación del talento y el autoconcepto en el rendimiento, incorporando, además, la influencia de las expectativas educacionales. Dado que el efecto de la interacción entre género y autoconcepto no resultó significativo, en los sucesivos modelos lo excluimos de los análisis. 
Tabla 2

Modelo Lineal Para SEPA de Lenguaje, Incluyendo el Autoconcepto Total Como Predictor (Beta No Estandarizado)

\begin{tabular}{|c|c|c|c|c|c|c|c|}
\hline Variables incluidas en el modelo & Modelo 1 & Modelo 2 & Modelo 3 & Modelo 4 & Modelo 5 & Modelo 6 & Modelo 7 \\
\hline Constante & $49,30^{*}$ & $48,99^{*}$ & $47,86^{*}$ & $47,14^{*}$ & $47,63^{*}$ & $35,29^{*}$ & $35,04^{*}$ \\
\hline Talento (sí) & $\begin{array}{c}6,81^{*} \\
(0,22)\end{array}$ & $\begin{array}{c}6,68^{*} \\
(0,22)\end{array}$ & $\begin{array}{c}6,63^{*} \\
(0,22)\end{array}$ & $\begin{array}{c}6,54^{*} \\
(0,22)\end{array}$ & $\begin{array}{c}6,47^{*} \\
(0,22)\end{array}$ & $\begin{array}{c}5,09^{*} \\
(0,17)\end{array}$ & $\begin{array}{c}5,08^{*} \\
(0,17)\end{array}$ \\
\hline Educación del padre (media o sup.) & & $\begin{array}{c}1,31^{*} \\
(0,07)\end{array}$ & $\begin{array}{c}0,68 \\
(0,04)\end{array}$ & $\begin{array}{r}0,63 \\
(0,03)\end{array}$ & $\begin{array}{c}0,60 \\
(0,03)\end{array}$ & $\begin{array}{r}1,01 \\
(0,05)\end{array}$ & $\begin{array}{c}1,01 \\
(0,05)\end{array}$ \\
\hline Educación de la madre (media o sup.) & & $\begin{array}{c}0,95 \\
(0,05)\end{array}$ & $\begin{array}{c}0,48 \\
(0,03)\end{array}$ & $\begin{array}{c}0,54 \\
(0,03)\end{array}$ & $\begin{array}{c}0,56 \\
(0,03)\end{array}$ & $\begin{array}{c}-0,21 \\
(-0,01)\end{array}$ & $\begin{array}{c}-0,21 \\
(-0,01)\end{array}$ \\
\hline Expectativas educacionales (educ. sup.) & & & $\begin{array}{c}2,82^{*} \\
(0,14)\end{array}$ & $\begin{array}{c}2,69^{*} \\
(0,13)\end{array}$ & $\begin{array}{c}2,72^{*} \\
(0,14)\end{array}$ & $\begin{array}{l}1,86^{*} \\
(0,09)\end{array}$ & $\begin{array}{l}1,86^{*} \\
(0,09)\end{array}$ \\
\hline Recursos en el hogar (PC) & & & & $\begin{array}{c}-0,40 \\
(-0,02)\end{array}$ & $\begin{array}{c}-0,42 \\
(-0,02)\end{array}$ & $\begin{array}{c}-0,14 \\
(-0,01)\end{array}$ & $\begin{array}{c}-0,14 \\
(-0,01)\end{array}$ \\
\hline Recursos en el hogar (espacio) & & & & $\begin{array}{l}1,55^{*} \\
(0,08)\end{array}$ & $\begin{array}{c}1,57^{*} \\
(0,08)\end{array}$ & $\begin{array}{c}1,01 \\
(0,05)\end{array}$ & $\begin{array}{c}1,01 \\
(0,05)\end{array}$ \\
\hline Sexo del alumno (hombre) & & & & & $\begin{array}{c}-1,03 \\
(-0,05)\end{array}$ & $\begin{array}{c}-0,11 \\
(-0,01)\end{array}$ & $\begin{array}{c}0,33 \\
(0,02)\end{array}$ \\
\hline Autoconcepto total & & & & & & $\begin{array}{l}0,14^{*} \\
(0,30)\end{array}$ & $\begin{array}{l}0,14^{*} \\
(0,31)\end{array}$ \\
\hline Interacción Autoconcepto-Sexo & & & & & & & $\begin{array}{c}-0,01 \\
(-0,02)\end{array}$ \\
\hline$R^{2}$ ajustado & 0,05 & 0,06 & 0,08 & 0,08 & 0,09 & 0,17 & 0,17 \\
\hline$F$ & 202,28 & 21,60 & 20,74 & 14,80 & 13,10 & 20,78 & 18,45 \\
\hline$N$ & 3816 & 974 & 908 & 907 & 907 & 787 & 787 \\
\hline
\end{tabular}

$* p<0,05$

Nota. Entre paréntesis hemos incluido los betas estandarizados.

En la Tabla 3 se observa la asociación del talento académico y las expectativas educacionales (educación superior) con el rendimiento académico de la prueba SEPA de Matemática. La educación de la madre (educación media o superior) también se relaciona; sin embargo, esta relación deja de ser significativa al incluir los recursos en el hogar. Al estimar el modelo con la variable autoconcepto como variable independiente (Modelo 6), las variables que también muestran una asociación significativa con el rendimiento en matemática son el talento y las expectativas educacionales, las que en conjunto explican un 14\% de la varianza de los puntajes de esta prueba. No se observan diferencias por sexo en la influencia del autoconcepto sobre los resultados de los alumnos en esta prueba. Estos resultados apoyan nuestra hipótesis sobre la relación entre el talento y el autoconcepto también en el rendimiento en matemáticas, controlando la asociación con las expectativas educacionales. 
Tabla 3

Modelo Lineal para SEPA de Matemática, Incluyendo el Autoconcepto Total Como Predictor (Beta No Estandarizado)

\begin{tabular}{|c|c|c|c|c|c|c|}
\hline Variables incluidas en el modelo & Modelo 1 & Modelo 2 & Modelo 3 & Modelo 4 & Modelo 5 & Modelo 6 \\
\hline Constante & $49,32^{*}$ & $47,95^{*}$ & $47,40^{*}$ & $46,81^{*}$ & $46,85^{*}$ & $35,82^{*}$ \\
\hline \multirow[t]{2}{*}{ Talento (sí) } & $6,06^{*}$ & $6,18^{*}$ & $6,00^{*}$ & $5,93^{*}$ & $5,92^{*}$ & $4,36^{*}$ \\
\hline & $(0,20)$ & $(0,21)$ & $(0,21)$ & $(0,21)$ & $(0,21)$ & $(0,16)$ \\
\hline \multirow[t]{2}{*}{ Educación del padre (media o sup.) } & & 0,41 & $-0,09$ & $-0,19$ & $-0,19$ & $-0,26$ \\
\hline & & $(0,02)$ & $(-0,01)$ & $(-0,01)$ & $(-0,01)$ & $(-0,01)$ \\
\hline \multirow[t]{2}{*}{ Educación de la madre (media o sup.) } & & $1,83^{*}$ & $1,30^{*}$ & 1,11 & 1,11 & 0,36 \\
\hline & & $(0,10)$ & $(0,07)$ & $(0,06)$ & $(0,06)$ & $(0,02)$ \\
\hline \multirow[t]{2}{*}{ Expectativas educacionales (educ. sup.) } & & & $2,00^{*}$ & $1,80^{*}$ & $1,80^{*}$ & $1,59 *$ \\
\hline & & & $(0,09)$ & $(0,09)$ & $(0,09)$ & $(0,08)$ \\
\hline \multirow[t]{2}{*}{ Recursos en el hogar (PC) } & & & & 1,04 & 1,04 & 1,19 \\
\hline & & & & $(0,06)$ & $(0,05)$ & $(0,06)$ \\
\hline \multirow[t]{2}{*}{ Recursos en el hogar (espacio) } & & & & 0,59 & 0,59 & 0,30 \\
\hline & & & & $(0,03)$ & $(0,03)$ & $(0,02)$ \\
\hline \multirow[t]{2}{*}{ Sexo del alumno (hombre) } & & & & & $-0,07$ & 0,51 \\
\hline & & & & & $(-0,01)$ & $(0,03)$ \\
\hline \multirow[t]{2}{*}{ Autoconcepto total } & & & & & & $0,12^{*}$ \\
\hline & & & & & & $(0,28)$ \\
\hline$R^{2}$ ajustado & 0,04 & 0,06 & 0,07 & 0,07 & 0,07 & 0,14 \\
\hline$F$ & 166,83 & 22,72 & 18,16 & 12,84 & 10,99 & 17,62 \\
\hline$N$ & 3976 & 1025 & 949 & 948 & 948 & 814 \\
\hline
\end{tabular}

$* p<0,05$

Nota. Entre paréntesis hemos incluido los betas estandarizados.

En el caso del grupo de niños no talentosos (ver Tabla 4), se observa que las expectativas académicas, los recursos en el hogar (espacio para estudiar) y ser mujer resultan ser importantes en el rendimiento que estos niños obtienen en la prueba de lenguaje (Modelos 3 y 4). De esta manera, cuando los padres tienen la expectativa de que sus hijos ingresen a la educación superior o los niños cuentan con un espacio adecuado para estudiar, el rendimiento en lenguaje es mejor. Cuando se incorpora la variable autoconcepto (Modelo 5), los recursos en el hogar y el sexo pierden importancia en su asociación con el rendimiento. En el grupo de niños no talentosos, las expectativas académicas y el autoconcepto explican un 13\% de la varianza de los puntajes de esta prueba. 
Tabla 4

Modelo Lineal Para SEPA de Lenguaje en Niños No Talentosos, Incluyendo el Autoconcepto Total Como Predictor (Beta No Estandarizado)

\begin{tabular}{|c|c|c|c|c|c|}
\hline Variables incluidas en el modelo & Modelo 1 & Modelo 2 & Modelo 3 & Modelo 4 & Modelo 5 \\
\hline Constante & $49,09^{*}$ & $47,87^{*}$ & $47,12^{*}$ & $47,75^{*}$ & $35,67^{*}$ \\
\hline Educación del padre (media o sup.) & $\begin{array}{c}1,22 \\
(0,06)\end{array}$ & $\begin{array}{c}0,60 \\
(0,03)\end{array}$ & $\begin{array}{c}0,51 \\
(0,03)\end{array}$ & $\begin{array}{c}0,49 \\
(0,03)\end{array}$ & $\begin{array}{c}0,84 \\
(0,04)\end{array}$ \\
\hline Educación de la madre (media o sup.) & $\begin{array}{c}0,81 \\
(0,04)\end{array}$ & $\begin{array}{c}0,32 \\
(0,02)\end{array}$ & $\begin{array}{c}0,35 \\
(0,02)\end{array}$ & $\begin{array}{c}0,37 \\
(0,02)\end{array}$ & $\begin{array}{l}-0,24 \\
(0,01)\end{array}$ \\
\hline Expectativas educacionales (educ. sup.) & & $\begin{array}{c}2,98^{*} \\
(0,15)\end{array}$ & $\begin{array}{c}2,83^{*} \\
(0,15)\end{array}$ & $\begin{array}{c}2,88^{*} \\
(0,15)\end{array}$ & $\begin{array}{l}1,74^{*} \\
(0,09)\end{array}$ \\
\hline Recursos en el hogar (PC) & & & $\begin{array}{c}-0,11 \\
(-0,01)\end{array}$ & $\begin{array}{c}-0,15 \\
(-0,01)\end{array}$ & $\begin{array}{c}0,01 \\
(0,00)\end{array}$ \\
\hline Recursos en el hogar (espacio) & & & $\begin{array}{c}1,45^{*} \\
(0,08)\end{array}$ & $\begin{array}{c}1,46^{*} \\
(0,08)\end{array}$ & $\begin{array}{c}0,93 \\
(0,05)\end{array}$ \\
\hline Sexo del alumno (hombre) & & & & $\begin{array}{c}-1,29 * \\
(-0,07)\end{array}$ & $\begin{array}{c}-0,47 \\
(-0,03)\end{array}$ \\
\hline Autoconcepto total & & & & & $\begin{array}{c}0,14^{*} \\
(0,31)\end{array}$ \\
\hline$R^{2}$ ajustado & 0,01 & 0,03 & 0,03 & 0,03 & 0,12 \\
\hline$F$ & 3,30 & 8,09 & 5,71 & 5,40 & 14,10 \\
\hline$N$ & 855 & 796 & 795 & 795 & 688 \\
\hline
\end{tabular}

$* p<0,05$

Nota. Entre paréntesis hemos incluido los betas estandarizados.

En el rendimiento de matemáticas de niños no talentosos se observa una mayor importancia de la tenencia de un computador en el hogar que de un espacio para estudiar (ver Tabla 5, Modelos 3 y 4). La educación de la madre tiene una asociación significativa con el puntaje en matemáticas (Modelo 1); sin embargo, al incluir las expectativas educacionales como variable independiente en el modelo (Modelo 2), esta asociación pierde su relevancia. Finalmente, controlando por las otras variables (Modelo 5), en el grupo de niños no talentosos únicamente la variable autoconcepto académico es capaz de explicar un 10\% de la varianza de los puntajes de esta prueba. 
Tabla 5

Modelo Lineal Para SEPA de Matemáticas en Niños No Talentosos, Incluyendo el Autoconcepto Total Como Predictor (Beta No Estandarizado)

\begin{tabular}{|c|c|c|c|c|c|}
\hline Variables incluidas en el modelo & Modelo 1 & Modelo 2 & Modelo 3 & Modelo 4 & Modelo 5 \\
\hline Constante & $48,13^{*}$ & $47,62^{*}$ & $46,93^{*}$ & $46,96^{*}$ & $35,86^{*}$ \\
\hline Educación del padre (media o sup.) & $\begin{array}{c}0,54 \\
(0,03)\end{array}$ & $\begin{array}{c}0,02 \\
(0,01)\end{array}$ & $\begin{array}{c}-0,13 \\
(-0,01)\end{array}$ & $\begin{array}{c}-0,13 \\
(-0,01)\end{array}$ & $\begin{array}{c}-0,26 \\
(-0,01)\end{array}$ \\
\hline Educación de la madre (media o sup.) & $\begin{array}{c}1,29^{*} \\
(0,07)\end{array}$ & $\begin{array}{c}0,81 \\
(0,04)\end{array}$ & $\begin{array}{c}0,62 \\
(0,03)\end{array}$ & $\begin{array}{c}0,63 \\
(0,03)\end{array}$ & $\begin{array}{c}-0,04 \\
(-0,00)\end{array}$ \\
\hline Expectativas educacionales (educ. sup.) & & $\begin{array}{l}1,91^{*} \\
(0,10)\end{array}$ & $\begin{array}{c}1,69^{*} \\
(0,09)\end{array}$ & $\begin{array}{c}1,69^{*} \\
(0,09)\end{array}$ & $\begin{array}{c}1,37 \\
(0,07)\end{array}$ \\
\hline Recursos en el hogar (PC) & & & $\begin{array}{c}1,27^{*} \\
(0,07)\end{array}$ & $\begin{array}{c}1,27^{*} \\
(0,07)\end{array}$ & $\begin{array}{c}1,11 \\
(0,06)\end{array}$ \\
\hline Recursos en el hogar (espacio) & & & $\begin{array}{r}0,65 \\
(0,03)\end{array}$ & $\begin{array}{c}0,65 \\
(0,03)\end{array}$ & $\begin{array}{c}0,43 \\
(0,02)\end{array}$ \\
\hline Sexo del alumno (hombre) & & & & $\begin{array}{l}-0,06 \\
(-0,01)\end{array}$ & $\begin{array}{c}0,43 \\
(0,02)\end{array}$ \\
\hline Autoconcepto total & & & & & $\begin{array}{c}0,12^{*} \\
(0,29)\end{array}$ \\
\hline$R^{2}$ ajustado & 0,01 & 0,01 & 0,02 & 0,01 & 0,10 \\
\hline$F$ & 3,16 & 4,20 & 3,58 & 2,98 & 12,04 \\
\hline$N$ & 896 & 825 & 824 & 824 & 706 \\
\hline
\end{tabular}

* $p<0,05$

Nota. Entre paréntesis hemos incluido los betas estandarizados.

En el caso del grupo de niños talentosos, el único factor que muestra alguna asociación con el rendimiento en la prueba de lenguaje es el autoconcepto (ver Tabla 6). Las expectativas de los padres - con una fuerte presencia en los niños no talentosos- no se destacan en este caso como un factor importante asociado al rendimiento en lenguaje. 
Tabla 6

Modelo Lineal Para SEPA de Lenguaje en Niños Talentosos, Incluyendo el Autoconcepto Total Como Predictor (Beta No Estandarizado)

\begin{tabular}{lccccc}
\hline Variables incluidas en el modelo & Modelo 1 & Modelo 2 & Modelo 3 & Modelo 4 & Modelo 5 \\
\hline Constante & $54,76^{*}$ & $54,47^{*}$ & $53,56^{*}$ & $53,15^{*}$ & $36,02^{*}$ \\
Educación del padre (media o sup.) & 1,86 & 1,00 & 1,06 & 1,12 & 2,54 \\
& $(0,10)$ & $(0,05)$ & $(0,06)$ & $(0,06)$ & $(0,14)$ \\
Educación de la madre (media o sup.) & 2,00 & 1,67 & 2,36 & 2,38 & $-0,25$ \\
& $(0,11)$ & $(0,09)$ & $(0,12)$ & $(0,13)$ & $(-0,01)$ \\
Expectativas educacionales (educ. sup.) & & 1,62 & 1,76 & 1,77 & 3,51 \\
& & $(0,08)$ & $(0,09)$ & $(0,09)$ & $(0,17)$ \\
Recursos en el hogar (PC) & & & $-2,73$ & $-2,81$ & $-1,78$ \\
& & & $(-0,15)$ & $(-0,15)$ & $(-0,10)$ \\
Recursos en el hogar (espacio) & & & $(0,12)$ & $(0,12)$ & $(0,09)$ \\
& & & & 1,06 & 2,74 \\
Sexo del alumno (hombre) & & & & $(0,06)$ & $(0,15)$ \\
& & & & & $0,15^{*}$ \\
Autoconcepto total & & & & $(0,26)$ \\
$R^{2}$ ajustado & & & & 0,078 \\
$F$ & 0,01 & 0,01 & 0,01 & 0,01 & 0,07 \\
$N$ & 1,75 & 0,94 & 1,15 & 1,01 & 2,00 \\
\hline
\end{tabular}

$* p<0,05$

Nota. Entre paréntesis hemos incluido los betas estandarizados.

Distinto es el escenario del grupo de niños talentosos en la prueba de matemáticas: además del autoconcepto, en la Tabla 7 es posible observar que la educación de la madre se asocia directamente con el rendimiento de estos niños, observándose que en presencia de una madre con educación media o superior, el niño presenta mejor calificación en SEPA de Matemáticas. No obstante, en presencia del autoconcepto (Modelo 5), este efecto pierde importancia y es esta última variable la que es capaz de explicar por sí sola un 5\% de la varianza en los puntajes de esta prueba. 
Tabla 7

Modelo Lineal Para SEPA de Matemática en Niños Talentosos, Incluyendo el Autoconcepto Total Como Predictor (Beta No Estandarizado)

\begin{tabular}{|c|c|c|c|c|c|}
\hline Variables incluidas en el modelo & Modelo 1 & Modelo 2 & Modelo 3 & Modelo 4 & Modelo 5 \\
\hline Constante & $52,24^{*}$ & $51,30^{*}$ & $51,58^{*}$ & $51,47^{*}$ & $40,27^{*}$ \\
\hline Educación del padre (media o sup.) & $\begin{array}{c}-0,44 \\
(-0,02)\end{array}$ & $\begin{array}{c}-0,68 \\
(-0,03)\end{array}$ & $\begin{array}{c}-0,71 \\
(-0,04)\end{array}$ & $\begin{array}{c}-0,70 \\
(-0,04)\end{array}$ & $\begin{array}{c}-0,29 \\
(-0,02)\end{array}$ \\
\hline Educación de la madre (media o sup.) & $\begin{array}{c}5,68^{*} \\
(0,28)\end{array}$ & $\begin{array}{c}4,63^{*} \\
(0,22)\end{array}$ & $\begin{array}{c}5,08^{*} \\
(0,25)\end{array}$ & $\begin{array}{c}5,09^{*} \\
(0,25)\end{array}$ & $\begin{array}{c}3,18 \\
(0,16)\end{array}$ \\
\hline Expectativas educacionales (educ. sup.) & & $\begin{array}{c}2,52 \\
(0,11)\end{array}$ & $\begin{array}{r}2,80 \\
(0,13)\end{array}$ & $\begin{array}{c}2,82 \\
(0,13)\end{array}$ & $\begin{array}{r}3,16 \\
(0,15)\end{array}$ \\
\hline Recursos en el hogar (PC) & & & $\begin{array}{l}-1,30 \\
(-0,07)\end{array}$ & $\begin{array}{c}-1,32 \\
(-0,07)\end{array}$ & $\begin{array}{c}0,94 \\
(0,05)\end{array}$ \\
\hline Recursos en el hogar (espacio) & & & $\begin{array}{c}-0,06 \\
(-0,00)\end{array}$ & $\begin{array}{c}-0,07 \\
(-0,00)\end{array}$ & $\begin{array}{c}-0,83 \\
(-0,04)\end{array}$ \\
\hline Sexo del alumno (hombre) & & & & $\begin{array}{r}0,25 \\
(0,01)\end{array}$ & $\begin{array}{c}1,24 \\
(0,06)\end{array}$ \\
\hline Autoconcepto total & & & & & $\begin{array}{c}0,10^{*} \\
(0,17)\end{array}$ \\
\hline$R^{2}$ ajustado & 0,06 & 0,05 & 0,04 & 0,03 & 0,05 \\
\hline$F$ & 5,03 & 3,29 & 2,04 & 1,70 & 1,88 \\
\hline$N$ & 128 & 123 & 123 & 123 & 107 \\
\hline
\end{tabular}

$* p<0,05$

Nota. Entre paréntesis hemos incluido los betas estandarizados.

\section{Discusión}

El talento académico está altamente asociado al autoconcepto académico del estudiante. Así, los niños con talentos académicos tienden a presentar un autoconcepto más alto que los niños sin talentos (Marsh \& Craven, 2006). Esto se observa también en los resultados que presentamos en este trabajo, en el que los estudiantes identificados como talentosos académicamente tienen un autoconcepto superior a sus pares identificados como no talentosos.

Estudios anteriores han mostrado que un adecuado autoconcepto académico permite un mejor rendimiento académico (Freeman, 2006) y que niños con talentos académicos suelen obtener mejores notas en el sistema escolar. Otros estudios demuestran que los alumnos conforman su autoconcepto académico en base a los resultados que obtienen en diferentes áreas y están constantemente retroalimentando la percepción de sí mismos (Marsh \& Craven, 2006). Nuestros resultados concuerdan con las investigaciones revisadas, ya que en los niños talentosos se observa una asociación entre talento y autoconcepto académico y ambos se relacionan con el rendimiento académico, tanto en matemáticas como en lenguaje. Otro factor relevante en su relación con el rendimiento académico son las expectativas de los padres de que sus hijos accedan a la educación superior.

De esta manera, nuestros resultados permiten identificar la relación entre expectativas académicas, autoconcepto y talento académico y cómo estos tres elementos conforman un círculo virtuoso que permite a alumnos obtener buenos resultados académicos, tanto en lenguaje como en matemáticas.

Lo interesante de estos resultados es la diferencia que existe entre niños talentosos y no talentosos en los factores que se asocian significativamente al rendimiento en pruebas estan- 
darizadas de lenguaje y matemáticas. Así, se observa que en niños talentosos el factor que se asocia casi exclusivamente a su rendimiento es el nivel de autoconcepto, perdiendo importancia factores como las expectativas de los padres, los recursos educativos, el sexo y la educación de los padres. Esto demuestra la importancia que toma el nivel del autoconcepto académico en niños talentosos para lograr buenos resultados académicos y, por lo tanto, el riesgo que podría producirse cuando el autoconcepto del niño talentoso es bajo.

Por el contrario, en niños no talentosos se observan variados factores que se asocian a su rendimiento, entre ellos, las expectativas de sus padres, la educación de los mismos, los recursos de apoyo a la educación que existen en el hogar y el autoconcepto. De esta manera, el autoconcepto en este grupo de niños es una variable que se suma a otras más y en conjunto a otros factores podrían influir sobre el logro académico.

La relevancia del rol del autoconcepto radica en la posibilidad que tiene de potenciar al máximo las habilidades innatas de los estudiantes (Cross \& Swiatek, 2009; Morawska \& Sanders, 2009). Nuestra investigación evidencia que un alto autoconcepto académico en niños con talentos ayuda a actualizar sus habilidades y a obtener buenos resultados académicos. Pero, al mismo tiempo, demuestra que un autoconcepto alto en niños sin talentos académicos también podría asociarse con la obtención de buenos resultados académicos.

Estos hallazgos deberán ser enriquecidos con futuras investigaciones que indaguen en la relación entre autoconcepto académico y los distintos tipos de inteligencia (analítica, práctica y creativa) que utilizamos en esta investigación para identificar a los niños con talentos académicos. La relación entre autoconcepto académico y talento y cómo estos influyen en los éxitos académicos posiblemente presenten distinciones entre niños con estos tres tipos de inteligencia.

Es importante hacer alusión a la dificultad con que nos encontramos para la comprensión de la relación que se establece entre la presencia de talentos académicos y las expectativas académicas. Estimar la influencia de las expectativas en el rendimiento académico es un desafío para futuras investigaciones, ya que las expectativas de los padres pueden ser conformadas en base a la identificación de talentos académicos en los hijos.

Por último, queremos destacar que estos hallazgos se observan en el contexto chileno y en una población específica de niños que asisten a colegios municipales de la comuna de Puente Alto, por lo que su generalización a otros contextos es limitada.

\section{Referencias}

Arancibia, V., Maltes, S. \& Álvarez, M. I. (1990). Test de autoconcepto académico. Santiago, Chile: Ediciones Universidad Católica de Chile.

Benito, Y. (1996). Inteligencia y algunos factores de personalidad en superdotados. Salamanca, España: Amarú.

Bloom, B. (1985). Developing talent in young people. New York, NY: Ballantine.

Bralic, S. \& Romagnoli, C. (2000). Niños y jóvenes con talentos. Una educación de calidad para todos. Santiago, Chile: Dolmen.

Brunel, M. L., Dupuy-Walker, L. \& Schleifer, M. (1990). Teachers' predictive capacity and empathy in relation to children's self-concept. BC Journal of Special Education, 14, 137-149.

Chan, D. W. (2005). Family environment and talent development of Chinese gifted students in Hong Kong. Gifted Child Quarterly, 49, 211-221. doi:10.1177/001698620504900303

Chile, Ministerio de Educación (2008). Matrículas 2008. Santiago, Chile: Autor. Extraído de http://www.mineduc.cl/

Chile, Ministerio de Planificación (2006). CASEN 2006. Santiago, Chile: Autor. Extraído de http://celade.cepal.org/ redatam/paises/chl/mideplanii/casen2006/Index.html

Colangelo, N. (2002). Counseling gifted and talented students. Iowa City, IA: The University of Iowa. Extraído del sitio web de The National Research Center on the Gifted and Talented, University of Connecticut: http://www.gifted. uconn.edu/nrcgt/newsletter/fall02/fall022.html

Colangelo, N. \& Davis, G. A. (2003). Introduction and overview. En N. Colangelo \& G. A. Davis (Eds.), Handbook of gifted education ( $3^{\mathrm{a}}$ ed., pp. 3-10). Boston, MA: Pearson Education.

Cross, T. L. \& Swiatek, M. A. (2009). Social coping among academically gifted adolescents in a residential setting: A longitudinal study. Gifted Child Quarterly, 53, 25-33. doi:10.1177/0016986208326554

Damon, W. \& Hart, H. (1982). The development of self-understanding from infancy through adolescence. Child Development, 53, 841-864. 
Demo, D. H. (1985). The measurement of self-esteem: Refining our methods. Journal of Personality and Social Psychology, 48, 1490-1502. doi:10.1037/0022-314.48.6.1490

Freeman, J. (2006, Noviembre). Un estudio de tres décadas sobre niños superdotados y talentosos. Ponencia presentada en el I Simposio Internacional Sobre Altas Capacidades Intelectuales, Las Palmas, España.

Gagné, F. (2003). Transforming gifts into talents: The DMGT as a developmental theory. En N. Colangelo \& G. A. Davis (Eds.), Handbook of gifted education ( $3^{\mathrm{a}}$ ed., pp. 60-74). Boston, MA: Pearson Education.

George, D. (1992). Gifted education in England. Roeper Review, 14, 201-204.

López, V. (2007). La inteligencia social: desde su estudio en niños y adolescentes con altas capacidades cognitivas. Psykhe, 16(2), 17-28. doi:10.4067/S0718-22282007000200002

Marsh, H. W. (2006). Self-concept theory, measurement and research into practice: The role of self-concept in educational psychology. London, Inglaterra: British Psychological Society.

Marsh, H. W. \& Craven, R. G. (2006). Reciprocal effects of self-concept and performance from a multidimensional perspective: Beyond seductive pleasure and unidimensional perspectives. Perspectives on Psychological Science, 1, 133-163. doi:10.1111/j.1745-6916.2006.00010.x

Marsh, H. W., Debus, R. \& Bornholt, L. J. (2005). Validating young children's self-concept responses: Methodological ways and means to understand their responses. En D. M. Teti(Ed.), Handbook of research methods in developmental psychology (pp. 138-160). London, Inglaterra: Blackwell.

Morawska, A. \& Sanders, M. R. (2009). Parenting gifted and talented children: Conceptual and empirical foundations. Gifted Child Quarterly, 53, 163-173. doi:10.1177/0016986209334962

Neihart, M., Reis, S. M., Robinson, N. M. \& Moon, S. M. (2002). The social and emotional development of gifted children: What do we know? Washington, DC: National Association for Gifted Children.

Núñez, J. C. \& González, J. A. (1994). Determinantes del rendimiento académico. Oviedo, España: Universidad de Oviedo.

Nurius, P. S. (1986). Reappraisal of the self-concept and implications for counseling. Journal of Counseling Psychology, 33, 429-438. doi:10.1037/0022-0167.33.4.429

Porter, L. (2006). Twelve myths of gifted education. Adelaide, Australia: Flinders University, School of Education. Extraído del sitio web de Louise Porter: http://www.louiseporter.com.au/pdfs/twelve_myths_of_gifted_education_ web.pdf

Preiss, D., Arancibia, V., Muñoz, B., Rosas, R., San Martín, E. \& Valenzuela, S. (2010a). Manual del Cuestionario de Vida Cotidiana para Niños y Niñas de $1^{\circ}$ a $4^{\circ}$ Básico. Santiago, Chile: Pontificia Universidad Católica de Chile, PENTA UC Escolar.

Preiss, D., Arancibia, V., Muñoz, B., Rosas, R., San Martín, E. \& Valenzuela, S. (2010b). Manual del Cuestionario de Creatividad para Niños y Niñas de $1^{\circ}$ a $4^{\circ}$ Básico. Santiago, Chile: Pontificia Universidad Católica de Chile, PENTA UC Escolar.

Preiss, D., Arancibia, V., Rosas, R. \& García, B. (2010). Sistema de identificación y selección de alumnos con talento académico de $1^{\circ}$ a $4^{\circ}$ básico (Proyecto FONDEF D05I10398). Santiago, Chile: Pontificia Universidad Católica de Chile, PENTA UC.

Purkey, W. W., Cage, B. N. \& Fahey, M. (1973). The Florida Key manual. Greensboro, NC: The University of North Carolina at Greensboro, Alliance for Invitational Education.

Renzulli, J. S. \& Reis, S. M. (2003). The schoolwide enrichment model: Developing creative and productive giftedness. En N. Colangelo \& G. A. Davis (Eds.), Handbook of gifted education (3 ${ }^{\mathrm{a}}$ ed., pp. 184-203). Boston, MA: Pearson Education.

Robinson, N. M., Lanzi, R. G., Weinberg, R. A., Ramey, L. S. \& Ramey, C. T. (2002). Family factors associated with high academic competence in former head start children at third grade. Gifted Child Quarterly, 46, 278-290. doi:10.1177/001698620204600404

Shavelson, R. J., Hubner, J. J. \& Stanton, G. C. (1976). Self-concept: Validation of construct interpretations. Review of Educational Research, 46, 407-441. doi:10.3102/00346543046003407

Shore, B. \& Kanevsky, L. (1993). Thinking processes: Being and becoming the gifted. En K. A. Heller, F. J. Mönks \& A. H. Passow (Eds.), International handbook of research and development of giftedness and talent (pp. 133-147). Elmsford, NY: Pergamon Press.

Silverman, L. (1997). Family counseling with the gifted. En N. Colangelo \& G. A. Davis (Eds.), Handbook of gifted education (pp. 382-397). Boston, MA: Allyn and Bacon.

Sternberg, R. J. (1997). A triarchic view of giftedness: Theory and practice. En N. Colangelo \& G. A. Davis (Eds.), Handbook of gifted education (pp. 43-53). Boston, MA: Allyn and Bacon.

Stipek, D. J. \& Tannatt, L. M. (1984). Children's judgments of their own and their peers' academic competence. Journal of Educational Psychology, 76, 75-84. doi:10.1037/0022-663.76.1.75

Tannenbaum, A. (2003). Nature and nurture of giftedness. En N. Colangelo \& G. A. Davis (Eds.), Handbook of gifted education ( $3^{\mathrm{a}}$ ed., pp. 45-59). Boston, MA: Pearson Education.

Terrasier, J. C. (1998). Les enfants surdoués [Niños superdotados]. Paris, Francia: ESF Editeur.

Valle, A., González, R., Núñez, J. C., Rodríguez, S. \& Piñeiro, I. (1999). Un modelo causal sobre los determinantes cognitivo-motivacionales del rendimiento académico. Revista de Psicología General y Aplicada, 52, 499-519.

Fecha de recepción: Julio de 2010.

Fecha de aceptación: Enero de 2012. 